\title{
Influencia del espesor óptico de aerosol en el índice ultravioleta (uv) basado con el modelo tropospheric ultraviolet and visible (tuv)
}

\section{Influence of aerosol optical thickness in the Ultraviolet (uv) index based with model tropospheric Ultraviolet and visible (tuv)}

\author{
Julio Ángeles, ${ }^{1}$ Roberto Angeles ${ }^{2}$
}

\section{RESUMEN}

Objetivos: Evaluar la influencia del espesor óptico de aerosol (EOA) en la radiación ultravioleta (RUV) que incide en la Estación Antártica Peruana Machu Picchu (ECAMP). Métodos: La investigación fue descriptiva comparativa. El principal instrumento para evaluar el EOA fue el fotómetro solar SP02-L para mediciones directas del sol con 4 sensores centrados en las longitudes de onda 412, 500, 675 y $862 \mathrm{~nm}$ Solo fueron utilizados el EOA a 500nm, como la distribución vertical de ozono, altura y el albedo de la ubicación. Con estos datos, el modelo generó datos cada minuto durante 24 horas durante el mes de enero de 2007, 2008 y febrero de 2013. Para su validación se utilizaron mediciones en tierra, sincronizándolo con el radiómetro espectral Ground-Based Ultraviolet (UV) GUV-511 Biospherical Inc., que brinda mediciones a 305, 320, 340 y $380 \mathrm{~nm}$. Se determinó el coeficiente de correlación de Spearman para la evaluación estadística, como la influencia del EOA en la radiación UV que incide en la Antártida. Resultados: Se determinó una máxima y mínima media de EOA de 0.1061 y 0.0616, respectivamente. Se reportó un coeficiente de correlación con el modelo TUV y el instrumento GUV de 0.8776 ( $p>0.05$ ) y se pudo validar el modelo TUV de la variación horaria de la radiación UV en esta ubicación polar, y por ende comprobar la influencia de los aerosoles disminuyen un 30\% del Índice UV por cada unidad de EOA. Conclusiones: Esto permitió implementar el modelo TUV para pronosticar la climatología anual de la RUV en la ECAMP como la influencia de los aerosoles en la RUV que incide en las regiones polares.

Palabra Clave: Espesor óptico de aerosol, radiación ultravioleta, Antártida.

\section{ABSTRACT}

Objectives: Evaluate the influence of Aerosol Optical Depth (AOD) in ultraviolet radiation (UVR) that affects the Peruvian Machu Picchu Station Antarctica (ECAMP). Methods: This research was a comparative descriptive. The main instrument for assessing the AOD was the SP02 - L sun photometer for direct measurements of the sun with 4 sensors centered at wavelengths $412,500,675$ and $862 \mathrm{~nm}$ only were used the AOD to $500 \mathrm{~nm}$ as the vertical distribution of ozone, height and location albedo. With these data, the model generated data every minute for 24 hours during the month of January 2007, 2008 and February 2013. For validation measurements used in land, synchronizing with the spectral radiometer Ground -Based Ultraviolet (UV) GUV - 511 Biospherical Inc., which provides measurement at 305, 320, 340 and $380 \mathrm{~nm}$. The Spearman correlation coefficient for statistical evaluation was determined as the influence of AOD in UV radiation reaching Antarctica. Results: The mean maximum and minimum of 0.1061 and

1. Ingeniero Ambiental, cargo: docente de la Universidad Alas Peruanas-filial Huancayo. email: j_angeles_s@doc.uap.edu.pe 2. Licenciado en Ciencias Fisicas,cargo: docente de la Universidad Alas Peruanas-filial Huancayo. email: roanvas@hotmail.com 
0.0616 of AOD, respectively were determined. A correlation coefficient with the TUV model and GUV of 0.8776 ( $p>0.05$ ) was reported instrument and could validate the TUV model the time variation of the UV radiation in the polar location, and thus test the influence of aerosols decrease to $30 \%$ of UV Index for each unit of AOD. Conclusions: This allowed implementing the TUV model to predict the annual climatology of UVR at ECAMP and the influence of aerosols on UV radiation incident on the Polar Regions.

Keyword: Aerosol optical depth, UV radiation, Antarctic.

\section{INTRODUCCIÓN}

Los aerosoles atmosféricos o material particulado, son un conjunto de partículas gaseosas, sólidas o líquidas suspendidas en la atmósfera (1), juegan un rol importante en el forzamiento climático de 2 maneras. Primero, la dispersión y absorción de la radiación solar que incide por los aerosoles que tiene una influencia directa en el balance radiativo de la atmosfera terrestre. Segundo, un efecto indirecto que es la formación de nubes (2).

Es tanto su importancia que la Organización Meteorológica Mundial (OMM) ha incluido el espesor óptico de aerosoles en el listado básico de medidas del Programa de Vigilancia Atmosférica Mundial ya que alteran en forma directa el clima debido a la dispersión y absorción de la radiación y la influencia en el albedo planetario en el sistema climático.

Los efectos indirectos incluyen la influencia de los aerosoles antropogénicos en núcleos de condensación para formación de nubes (3). Y la presencia de los aerosoles incrementa la concentración de los núcleos de condensación y consecuentemente incrementa la absorción de la radiación ultravioleta (UV) del tipo B. Una estimación del espesor óptico de aerosol (EOA) reduce la irradiancia UV-B alrededor de 5 a 35\% (4).

Estudios anteriores indicaron que los aerosoles absorben radiación UV-B y afecta de esta manera la cantidad recibida de radiación UV por los humanos y la biosfera (5). Esto es muy importante para cuantificar la absorción en el UV-B por los aerosoles como la intensidad de UV-B que afecta la salud directamente a los humanos, por ejemplo, a través del cáncer de piel (6).

El problema que conduce al presente trabajo es: ¿Cuál es la influencia del espesor óptico de aerosol en la radiación UV que incide en la Estación Antártica Peruana Machu Picchu(ECAMP) en los meses de enero del 2007, 2008 y febrero de 2013 ?

El objetivo es evaluar la influencia del espesor óptico de aerosol en la radiación UV que incide en la Estación Antártica Peruana Machu Picchu.

Con la investigación se busca determinar la influencia de los aerosoles en la radiación UV que incide a la superficie a cielo despejado y contribuir a implementar modelos climatológicos y a reducir riesgos de un problema de salud pública como es el cáncer de piel.

\section{MÉTODOS Y MATERIALES}

Las mediciones se dieron en la estación científica Antártica Peruana Machu Picchu (ECAMP) ubicada en la Punta Crespín, Ensenada Macke-

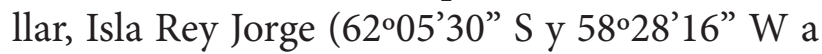
6 m.s.n.m) durante los meses de enero y febrero para los años 2007(ANTAR XVII), 2008(ANTAR XVIII) y 2013 (ANTAR XXI).

Para la determinación del espesor óptico de aerosol (EOA), se utilizó un fotómetro solar SP02-L (Middleton Inc. Australia) (7); este instrumento es utilizado para observaciones de aerosoles, ozono y vapor de agua. Para la configuración del aerosol, el SP02-L tiene 4 canales en las longitudes de onda de 412, 500, 675 y 862nm, corres- 
pondientes a la radiación visible. Estos espectro-piroheliometros se encuentran axialmente alineados en un recinto herméticamente cerrado, los mismos que actúan de modo simultáneo cuando el instrumento es alineado directamente al sol, acción que permite una resolución fina de indicación del espesor óptico de aerosol en la atmósfera.

La versión SP02-L tiene un ancho de banda de $10 \mathrm{~nm}$ y un campo de visión de $2.5^{\circ}$, las señales de salida tienen un rango de -0.05 a 4.50 VDC, adicionalmente tiene una salida de temperatura interna $\left(10 \mathrm{mV} / 1^{\circ} \mathrm{C}\right)$. La temperatura de operación del fotómetro solar es de $-30^{\circ} \mathrm{C}$ a $70^{\circ} \mathrm{C}$.

El modelo radiativo utilizado en el presente trabajo de evaluación de la radiación UV fue estimado por el modelo tropospheric ultraviolet and visible (TUV) del National Center for Atmospheric Research (NCAR) versión $4.1^{\text {a }}(8)$. Este es un modelo radiativo usado sobre el rango de radiación de 121 a 750nm, para calcular la irradiancia espectral, el flujo actínico, los coeficientes de fotodisociación (valores J), y la irradiancia efectiva biológicamente, y a partir de esta el índice UV.

El código está escrito en fortran 77 estándar y opera en un entorno UNIX. Este modelo fue adaptado a las condiciones de la Antártida, habiéndose cambiado valores característicos de la ubicación.

La distribución temporal del ozono fue obtenido con el OMI con un promedio de 291.86 UD, con una desviación estándar de \pm 16.66 UD. En este caso, se consideró la influencia del EOA y otros contaminantes del aire; pero no fue considerado la nubosidad debido a su alta variabilidad y difícil capacidad de pronóstico. La variable principal de estudio generada por el modelo fue el índice UV para determinar cómo influye el EOA en el índice UV.

El índice UV se reporta como un valor entero de 0 a 20 e indica niveles de RUV eritemica sobre la superficie terrestre y permite a las personas cal- cular su tiempo máximo de exposición al sol sin riesgo a sufrir un eritema.

Para la medición espectral de la RUV se utilizó el radiometro de filtro multicanal GUV-511, fabricado por Biospherical Instrument Inc. (BSI), San Diego, California, EE.UU. El radiómetro tiene 4 canales en la región UV, centrados en 305, 320, 340 y $380 \mathrm{~nm}$, respectivamente. El ancho de banda espectral de cada uno es aproximadamente de 10nm (full-width at half-maximum, FWHM). Este instrumento fue instalado libre de interferencias en la ECAMP.

Cada uno de los 4 canales fue sincronizado para registrar promedios de la irradiancia, cada minuto, medidos en unidades de uW.cm-2nm-1 (para convertir a Wm-2.nm-1 solo se divide entre 100). En el caso del radiómetro GUV-511, para la obtención del I-UV los valores de 305, 320 y $340 \mathrm{~nm}$ son multiplicados con sus respectivos valores de calibración para luego sumarlos y finalmente, obtener el valor del I-UV.

El registro de mediciones con este instrumento se realiza desde setiembre del 2002 y ha venido siendo realizado tanto en Huancayo como en la ECAMP con apoyo de las universidades locales y del Instituto Antártico Peruano. Es el único medidor espectral de radiación UV del Perú. Finalmente, para el análisis de datos se determinó el coeficiente de correlación de Spearman a un nivel de confianza de $95 \%$.

\section{Espesor óptico de aerosol (EOA)}

El EOA como se indica en la ecuación 1 se estima a partir del espesor óptico total $(\tau)$, el cual es la suma de los espesores ópticos del aire (referido como dispersión Rayleigh, ( $\tau$ Rayleigh),

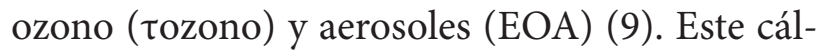
culo se determina para cada una de las longitudes de onda correspondientes al fotómetro solar SP02-L, siendo 412, 500675 y $862 \mathrm{~nm}$.

$$
E O A=\tau-\tau_{\text {Rayleigh }}-\tau_{\text {ozono }}
$$


La secuencia de ecuaciones se basa en el cálculo de los tres primeros espesores ópticos. La determinación del espesor óptico total está basada en la Ley de Lambert-Beer (ecuación 2), que explica la atenuación de la radiación solar al paso por la atmósfera (10).

$$
\tau=\frac{\mathbf{h}\left(\mathbb{R}_{0 \lambda} / I_{\lambda}\right)}{m}
$$

Donde $\mathrm{R}$ es el factor de corrección distancia sol-tierra; $I \_\lambda$ es la intensidad de radiación observada a una longitud de onda $(\lambda)$, a nivel de la superficie del suelo; I_o $\lambda$ es la intensidad de radiación en el tope de la atmósfera, obtenido gráficamente por el método de Langley (11), para una $\lambda$ en particular; $y$ m es la masa del aire determinado por la secante del ángulo cenit solar.

El método de Langley está basado en el principio de extinción espectral y la ley de Beer- LambertBouguer. En este método las observaciones de radiación son ploteados como una función de la masa de aire. La pendiente de la regresión lineal resultante es ( ttotal) y la radiación correspondiente a $m$ igual a cero es la I_o $\lambda$.

El espesor óptico del aire, conocido como el de Rayleigh, es calculado utilizando la ecuación 3 (12):

$$
\tau_{R}(\lambda)=(a+b H) \lambda^{-\left(c+d \lambda+\frac{b}{\lambda}\right)} \frac{P}{P_{s}}
$$

Donde, $a=0.00864, b=6.5^{\star} 10-6, c=3.916, d=0.074$, $\mathrm{e}=0.05 ; \mathrm{H}$ es la altura del fotómetro solar SP02-L en kilómetros; P es la presión de sitio ( 986 en $\mathrm{mb})$; Ps, presión atmosférica estándar 1013.25 $\mathrm{mb} ; \mathrm{y} \lambda$ la longitud de onda en micrómetros (12). El espesor óptico de ozono está en función de la concentración de la columna total de ozono $(\bigotimes)$ en Unidades Dobson obtenido con el sensor OMI y el coeficiente de absorción de ozono $(\mathbb{\nabla})$ según la ecuación 4 .

$$
\tau_{O 3}=\frac{\Omega \times \sigma}{1000}
$$

Para las longitudes de onda de 412, 500, 675 y 862, los coeficientes de absorción son $0.000295974375,0.031997375,0.0401001875 \mathrm{y}$ 0.00247309125 , respectivamente $(14=13)$.

\section{RESULTADOS}

\section{Variación de la Radiación UV y validación del mo- delo TUV}

Se generaron datos del I-UV con el modelo TUV cada minuto durante 24 horas por 2 meses (Enero y Febrero) generándose dos matrices de $1440 * 59$ datos.

Una muestra de los resultados se presenta en la figura N0 1, que son mediciones efectuados en verano en el continente antártico, para los días más despejados.

Es posible ver la adecuada replica de la variación horaria de la RUV, pero además la diferencia debido a la presencia de nubes.

También estos resultados se presentan considerando las recomendaciones de especialistas para uniformizar el reporte del índice UV(14), que indican que cuando se pronostica el índice UV del día siguiente este es elaborado para el medio día y asumiendo el cielo despejado, y también que el rango de incertidumbre puede estar entre $+-10 \%$.

Utilizando el software Matlab para realizar la prueba estadística de correlación de Pearson entre las mediciones en tierra y datos del modelo dando un coeficiente de correlación de 0.8776 $(\mathrm{p}<0.05)$.

Con ello se asume que el valor es aceptable y así emplear el modelo TUV para modelar la RUV en la ECAMP considerando el material particulado $\mathrm{o}$ aerosoles. 
Figura $\mathrm{N}^{\circ}$ 1: Variación de la radiación UV con el equipo GUV-511 y el modelo TUV

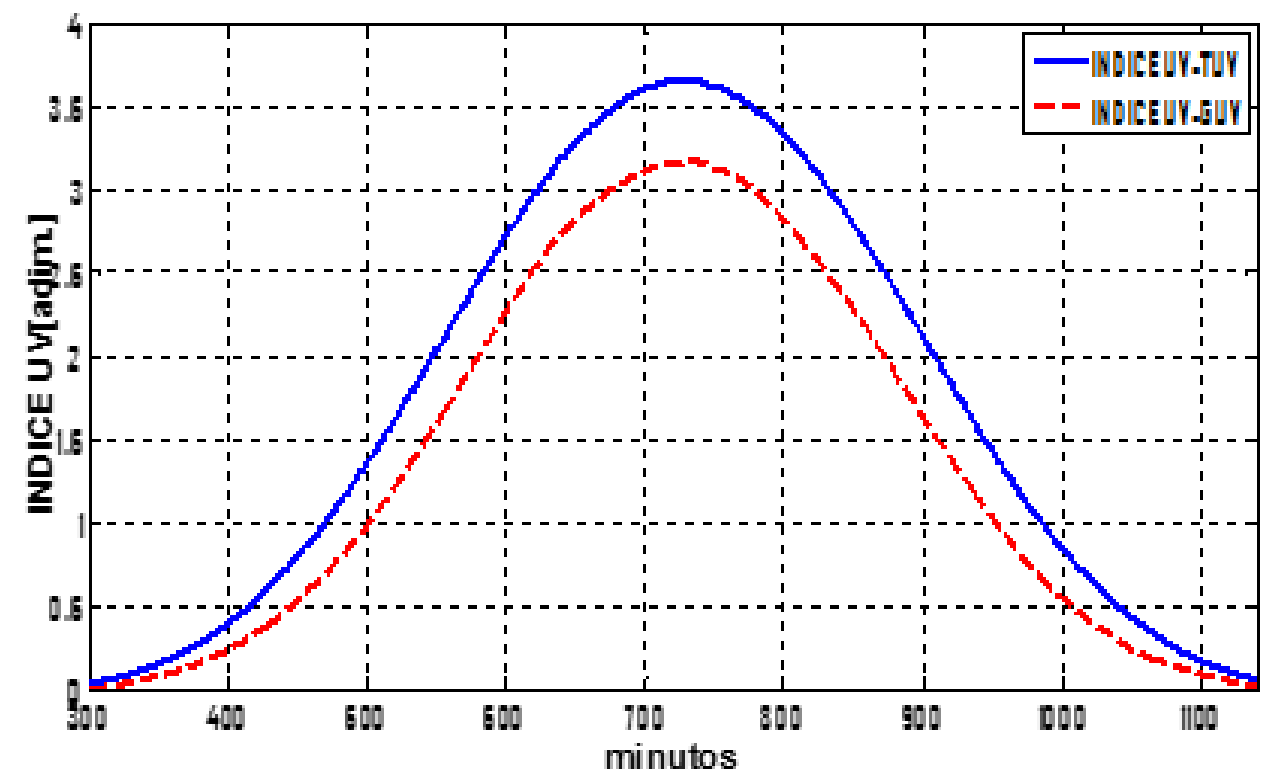

\section{Espesor óptico de aerosol}

En la figura $\mathrm{N}^{\circ} 2$ muestra una serie histórica de los valores del AOD(500nm) en la ECAMP 2007, 2008 y 2013. Como se puede observar el $\mathrm{AOD}(500 \mathrm{~nm})$ varía entre 0.0646 a 0.1061 , el $\mathrm{AOD}(862 \mathrm{~nm})$ varía entre 0.0391 a 0.1039 , siendo típico por las condiciones de turbidez atmosférica del sitio en este tiempo del año(15). La campaña ha sido planificada con el principal objetivo de cuantificar la comparación que existe entre los distintos años de mediciones. En el primer paso, se ha requerido los valores del espesor óptico de Rayleigh(ROD), espesor óptico de ozono(OOD) y SZA usado en el análisis de datos. Se ha verificado que: (a) ROD asume valores de 0.1154 a 500nm, (b) los valores de OOD a 500nm es de 0.0085 .

Figura N²: Variación diaria del AOD a 500 nm para los años de 2007, 2008 y 2013

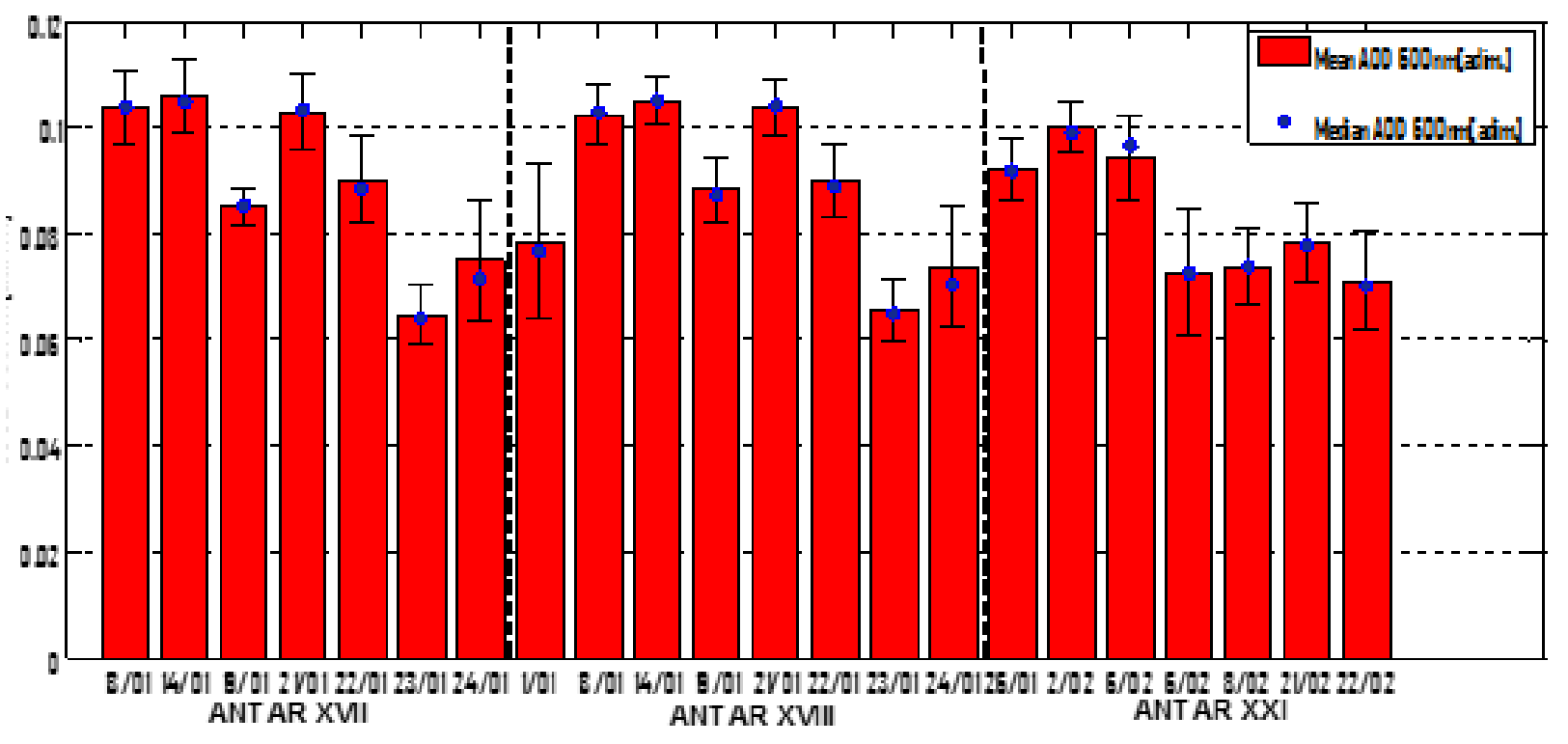


Estos resultados de espesor óptico de aerosol (AOD) están dentro del rango de los hallados en regiones polares por otros grupos (16), siendo menores en relación con los encontrados en ciudades urbanas entre 0.25 a 1.7 (17) y mucho menores que los registrados en lugares de quema de la biomasa donde llegan a valores en el rango de 0.6 a 2.4 para similares longitudes de onda (18).

\section{Influencia del EOA en el IUV}

Los datos experimentales revelan una disminución de 30\% de índice UV obtenidos con el modelo TUV por unidad de EOA durante el mes de enero y febrero del 2013 en la ECAMP. Los cálculos del EOA se realizaron sin cobertura de nieve y en condiciones normales correspondientes a días despejados.

La obtención de valores muy altos de índice UV (IUV) para días de cielo claro y en horas cercanas al mediodía solar, se debe al bajo EOA considerado por el modelo TUV, en cambio los bajos valores de IUV indican la alta presencia de aero- soles, como indicador el EOA considerado en el modelo TUV.

Las definiciones sobre el índice UV se encuentran en el texto, índice UV solar mundial: guía práctica, preparada durante varios años de trabajo por un grupo de especialistas y es una recomendación conjunta de la organización mundial de salud(OMS), la organización meteorológica mundial(OMM), el programa de las naciones Unidad para el medio ambiente (PNUMA) y la comisión Internacional de Protección contra la radiación no Ionizante(ICNIRP) donde se describen las estrategias básicas para la difusión adecuada del IUV(19).

Figura $\mathrm{N}^{\circ}$ 3: Variación del índice UV respecto al EOA

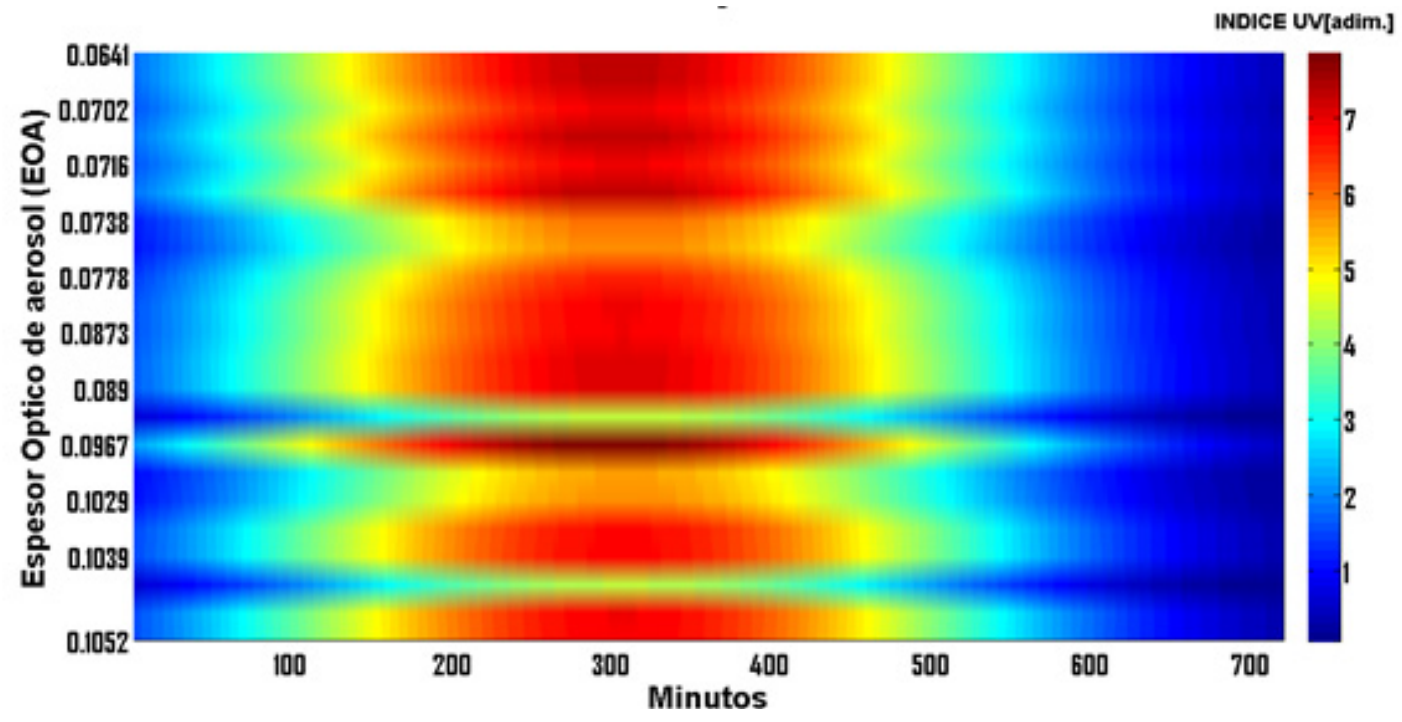

\section{DISCUSIÓN}

Los modelos no pueden ser más precisos que datos de entrada que se usan. Las incertezas en los datos espectrales (irradiancias espectrales extraterrestres, secciones transversales de absorción, etc), en la composición de la atmósfera (ozono, nubes, etc.), y en la topología y reflectividad de la superficie, son todos los grandes componentes de cualquier error de los modelos utilizados.
Se introducen incertezas adicionales debido a la necesidad de eficiencia computacional. Estos pueden incluir varias aproximaciones a la verdadera solución de la ecuación de transferencia radiativa. Resulta difícil decidir, en general, si las aproximaciones que resultan de la modelización son mayores que las incertezas que resultan de los datos de entrada. 
La experiencia de los últimos años ha mostrado que si el estado del cielo está extremadamente bien caracterizado (libre de nubes, libre de contaminantes, con medidas auxiliares del perfil de ozono, de la reflectibidad superficial, etc) (20).

En el cual estudios similares una estimación del espesor óptico de aerosol (EOA) reduce la irradiancia UV-B alrededor de 5 a 35\% (4). En que indicaron que los aerosoles absorben radiación UV-B y afecta de esta manera la cantidad recibida de radiación UV por los humanos y la biosfera (5).Y La correlación en estudios similares entre índice UV y EOA en abril es de 0.86 en Nepal (21)

En cambio en nuestra investigación el impacto de la variación del EOA en la cantidad recibida de UV-B en la superficie tomando como indicador el índice UV.

Indica que debido a la presencia de aerosoles el índice UV disminuye en un $30 \%$, por lo tanto altera el balance radiativo directo de los aerosoles polares en la superficie y en la parte superior de la atmosfera que necesitan ser estudiados más detenidamente a través de estudios teóricos sobre las propiedades radiativas de los aerosoles(22).

\section{REFERENCIAS BIBLIOGRÁFICAS}

1. Vergaz B. Propiedades ópticas de los aerosoles atmosféricos. Caracterización del área del Golfo de Cádiz, Valladolid. [Tesis Doctoral]: Universidad de Valladolid; 2001.

2. Foster P, Ramaswamy. IPCC, Changes in atmospheric constituents and in radiative forcing. UNIVERSITY PRESS CAMBRIDGE. NEW YORK, USA; 2007.

3. WMO. Report of the WMO.WMO aerosol measurement procedure; 2003

4. Kylling A., A. F. Bais, M. Blumthaler, J. Schreder, C. S. Zerefos and E. Kosmidis, Effect of aerosols on solar UV irradiances during the photochemical activity and solar ultraviolet radiation campaign, J. Geophys. Res. (103), (D20), 26,051- 26,060, 1998.

5. Wenny, B. N., J. S. Schafer, J. J. de Luisi, W. K. Saxena, W. F. Barnard, I. V. Petropavlovskih, A. J. Vergamini, A study of regional aerosol radiative properties and effects on ultra- violet- B radiation, J. Geophys. Res. (103), 17,083-17,097, 1998.

6. Diffey, B. L., Solar ultraviolet radiation effects on biological systems, Phys. Med. Biol., 36, 299-328, 1991.

7. Guía de usuario del fotómetro solar modelo SP02-L de Middleton Imc; 2004

8. Michalsky J. Aerosol optical depth value-added product. Climate Research. 2013; 129: 1-32.

9. Bodhaine B, Wood N, Dutton E, and Slusser J. On rayleigh Optical Depth Calculations. Journal Atmos. and Ocean. 1999; 16: 1854-1864.

10. Tomasi C, Vitale V. Calculation of the relative optical mass functions for air, water vapor, ozone and nitrogen dioxide in the Antarctic and Arctic Atmospheres. 7th Workshop Italian Research on Antarctic Atmosphere, Conference Proceedings. Italian Physical Society, Bologna, Italy. 1997; 62: 22-24.

11. Liou K. An introduction to atmospheric radiation. 2da ed. New York: academic Press; 2007.

12. Reagan J, Scott - Fleming I., Herman BySchotland R.Recovery of spectral optical, depth and zero - air mass solar spectral irradiance under conditions of temporally varying optical depth proceedings of IGARSS'84 Symposium. Strasbourg. 1984; 215: 455-459.

13. MADRONICH S. UV radiation in the natural and perturbed atmospher, en M. Tevini, (editor), 'Environmental Effects of UV(ultraviolet) radiation", Lewis Publisher, Boca Raton, pags. 17-69, 1993.

14. WMO. Report of the WMO-WHO Meeting of experts on standardization of UV índices and their disseminaton to the public, LesDiablerets, Rep. 127; 1998.

15. Dutton E, Reddy P, Ryan S, Tomasi C. Aerosol in polar regions; A historical overview based on optical depth and in situ observations, J. Geophys. Res. 2007; 112: 1-28.

16. Stone R. Monitoring aerosol optical depth at Barrow, Alaska and South Pole; Historical overview, recent results, and future goals. Coop. Res. in Environ. Scien. 2002; 80: 123-144.

17. Castro T, Madronich S, Rivale S, Muhlia A, Mar B. influence of aerosols on photochemical smog in Mexico City. Atmospheric Environment. 2001; 35: 1765-1772.

18. Eck T, Holben B, Reid J, O’Neill N, Schafer J, Dubovik O, Simimov A, Yamasoe M y Artaxo P. High aerosol optical depth biomass burning events: A comparison of 
optical properties for different source regions. Geophysical Research Letters. 2003; 30(20): 2035-2044.

19. WHO. Global SolarUV Index: a practical guide, WHO/ SDE/OEH/02.2, disponible en español en: http://www. Who.in/uv/.publicaions/en/uvispa.pdf, Ginebra, Suiza; 2002.

20. Torres M, Saravia L. Modelización de la radiación solar ultravioleta: aplicación y testeo del TUV. ASADES. 2001; 5:35-40.

\section{Anexos:}

21. Niranjan S, Binod K. Satellite estimation and ground measurements of solar UV index and influence of aerosol in ground based UV data in Kathmandu, Nepal. Environ. Science. 2013; 5:1362-1372.

22. Mazzola M, Stone R. Evaluation of sun photometer capabilities for retrievals of aerosol optical depth at high latitudes: The POLAR-AOD intercomparison campaigns. Atmos. Environ. 2012; 52:4-17.

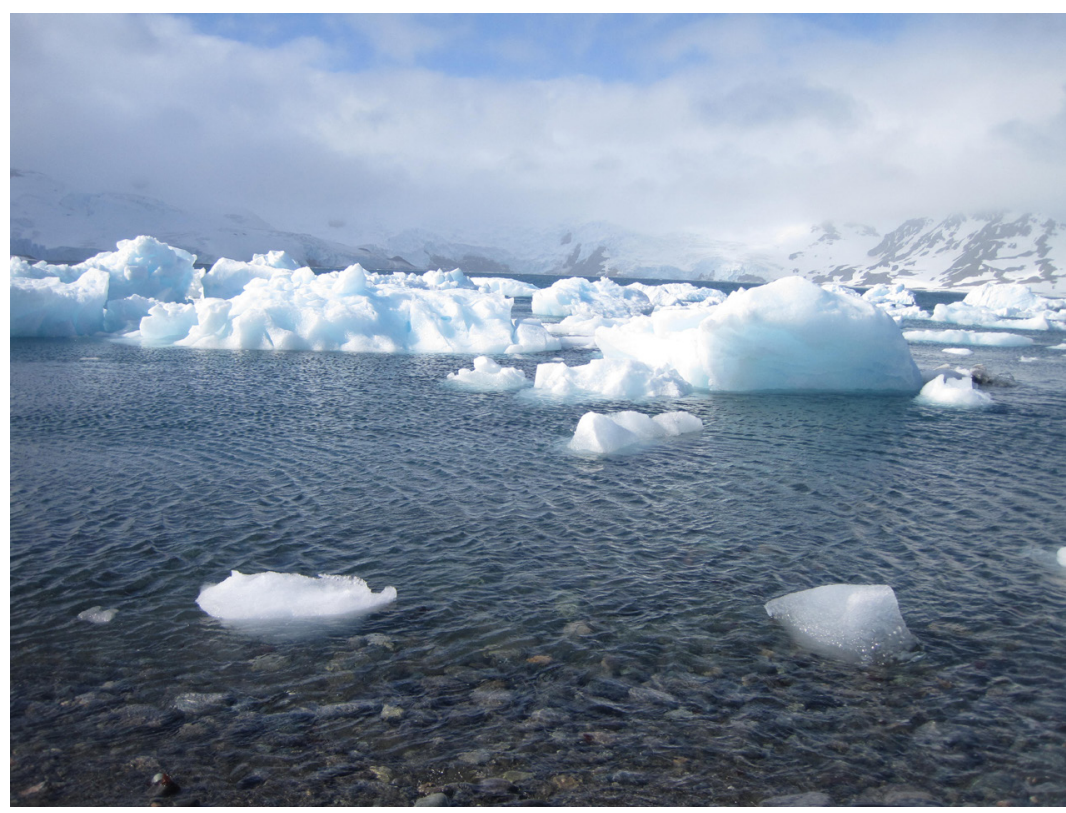

Estación Antártica Peruana Machu Picchu

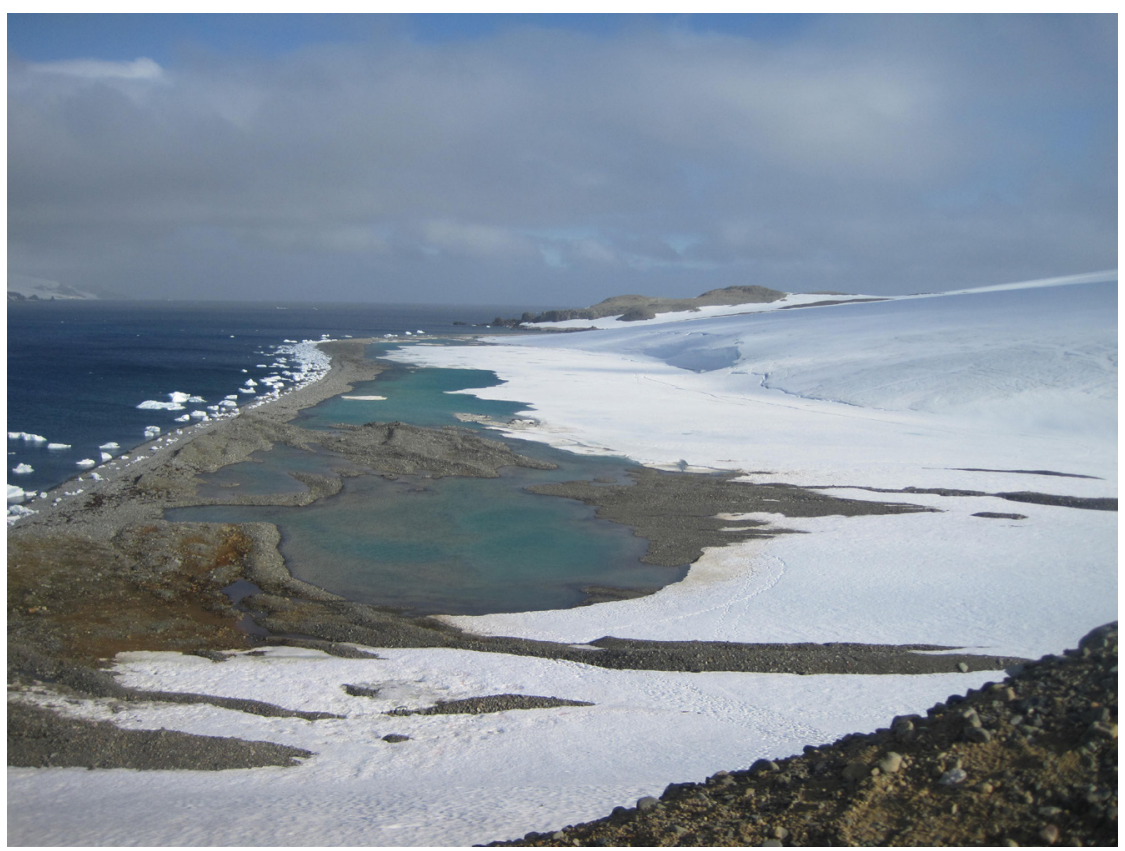

Masa Glaciar en la Antártida 